ISSN 1392-3196 / e-ISSN 2335-8947

Zemdirbyste-Agriculture, vol. 102, No. 1 (2015), p. 95-102

DOI $10.13080 /$ z-a.2015.102.012

\title{
The effect of different types of inoculants on the characteristics of alfalfa, ryegrass and red clover/ryegrass/timothy silage
}

\author{
Jonas JATKAUSKAS ${ }^{1}$, Vilma VROTNIAKIENE ${ }^{1}$, Anouk LANCKRIET ${ }^{2}$ \\ ${ }^{1}$ Institute of Animal Science, Lithuanian University of Health Sciences \\ R. Žebenkos 12, Baisogala, Radviliškis distr., Lithuania \\ E-mail: lgipts@gmail.com \\ ${ }^{2}$ DeLaval NV \\ Drongen, Belgium
}

\begin{abstract}
An in vitro laboratory study using $3 \mathrm{~L}$ mini silos was conducted in 2012-2013 to observe the effects of 8 inoculants on silage nutrient composition, $\mathrm{pH}$, volatile fatty acids concentration, dry matter loss, and aerobic stability. Alfalfa (Medicago sativa L.) in early-flower stage (first harvest, $355 \mathrm{~g} \mathrm{~kg}^{-1} \mathrm{DM}$ ), perennial ryegrass (Lolium perenne L.) in early-boot stage (first harvest, $322 \mathrm{~g} \mathrm{~kg}^{-1} \mathrm{DM}$ ) and mixture of red clover (Trifolium pratense L.), perennial ryegrass (L. perenne) and timothy (Phleum pratense L.) at a ratio 60:25:15 of fresh matter, in early bloom stage of red clover (first harvest, $343 \mathrm{~g} \mathrm{~kg}^{-1} \mathrm{DM}$ ) were used. The trials were conducted as a randomized complete block design, with five replicates per crop per treatment. Each trial had 9 treatments (uninoculated control and 8 inoculants Feedtech products (F10, F18, F22 and F3000), and products Sil-All $4 \times 4$ (ISA), Lalsil Dry (LD), Bonsilage (IBO) and Bio-sil Stabil (BS), with 5 silos per treatment. The mini silos were filled to a target density of $0.2 \mathrm{~kg} \mathrm{~L}^{-1} \mathrm{DM}$. After a 90-day storage at $20^{\circ} \mathrm{C}$, silages were sampled and analyzed. All the eight microbial inoculant products improved many aspects of silage quality across the range of silage analytes examined and across the three forage types tested. Inoculant treated alfalfa, perennial ryegrass and red clover/ryegrass/timothy silage had significantly lower $\mathrm{pH}$ values, ammonia-N concentration and dry matter losses, but significantly higher lactic acid concentration compared to untreated silage. Inoculants F18, F22, F3000 and ISA gave significantly lower pH value than F10, $\mathrm{LD}, \mathrm{IBO}$ and $\mathrm{BS}$ in alfalfa silage and in perennial ryegrass silage. The $\mathrm{pH}$ value for the red clover/ryegrass/timothy silage treated with inoculants F10, F22 and F3000 was significantly lower compared to F18, ISA, LD, IBO and BS inoculants treated silages. Lactate was significantly higher in perennial ryegrass silage for F18 and F22 inoculants compared with F10, F3000, ISA and LD inoculants and in red clover/ryegrass/timothy silages for F10, F22, F3000 and ISA inoculants versus $(v s)$ F18, LD, IBO and BS inoculants. Dry matter loss was lower $(P<0.05)$ in perennial ryegrass silages for F10, F18, F22 and F3000 inoculants compared with ISA, LD and IBO inoculants, and in red clover/ryegrass/timothy silages for F10, F18, F3000 and ISA inoculants compared with F22, LD, IBO and BS inoculants $(4.02 \%$ vs $4.18 \%, P<0.05)$. Addition of the inoculants improved aerobic stability of alfalfa silages by $126-204 \mathrm{~h}$, of perennial ryegrass silages by $18-216 \mathrm{~h}$ and of red clover/ryegrass/timothy silages by $30-138 \mathrm{~h}$ compared to the uninoculated control. The results suggest that the lactic acid bacteria inoculants were effective in improving the silage quality in moderately difficult and difficult to ensile forage.
\end{abstract}

Key words: aerobic stability, fermentation, lactic acid bacteria.

\section{Introduction}

Ensiling is a preservation method for moist forage crops based on converting water soluble carbohydrates into organic acids, mainly lactic acid, and preventing high ammonia nitrogen levels in anaerobic conditions by lactic acid bacteria. Silage quality and nutrient use efficiency are influenced by a number of factors such as crops, ensiling technologies, machinery and additives used for manipulating fermentation processes (Davies et al., 2005). The biological additives were evaluated for their potential to improve silage fermentation and nutritive value (Filya et al., 2007) and for their ability to increase animal productivity (Contreras-Govea et al., 2009).
Homo-fermentative bacteria convert C6-sugars solely into lactic acid, whereas hetero-fermentative species produce lactic acid and carbon dioxide at equal shares as well as traces of acetic acid or ethanol. Wellknown species of the homo-fermentative bacteria are Lactobacillus plantarum, Pediococcus acidilactici, P. pentosacceus, Enterococcus faecium, L. delbrueckii, L. casei and L. rhamnosus. Commonly available heterofermentative bacteria are L. brevis and L. buchneri. To this day, we have the knowledge how microorganisms affect silage quality and the dynamics of microbial populations at ensiling time. However, there is still little information available on how significant the new 
species are to silage preservation (Muck, 2012). There are different objectives for using silage additives. The main objectives are to improve fermentation, reduce dry matter loss and to prevent secondary fermentation (to increase aerobic stability) after opening of the silo. The Deutsche Landwirtschafts-Gesellscaft Guidelines for the testing silage additives take into account the principal inoculums action, which are improving the fermentation process on the one hand and improving aerobic stability on the other hand (Kleinschmit et al., 2005; Weib et al., 2011). The companies producing inoculants expect that new strains and mixtures will be highly competitive and improve silage fermentation by reducing $\mathrm{pH}$ and by producing largely lactic acid, compared to spontaneously fermented silage. However, some authors reported that homo-fermentative lactic acid bacteria inoculants did not improve the aerobic stability of silages (Sucu, Filya, 2006). After opening of the silo, yeast and moulds can lead to an increase in $\mathrm{pH}$ value and temperature of the silage as well as to a reduction of readily available sugars. Loss of carbon dioxide and temperature increase cause dry matter losses and reduce the feeding value of silage (Muck, 2012). The present study was conducted in order to assess which mixtures of lactic acid bacteria have a greater potential to improve fermentation pattern of the alfalfa, perennial ryegrass and red clover/ryegrass/timothy silage and whether inoculants have an effect on the extent of deterioration occurring during the exposure to air.

\section{Material and methods}

Mini silo experiments. Alfalfa (Medicago sativa L.) in early-flower stage (first harvest), perennial ryegrass (Lolium perenne L.) in early-boot stage (first harvest) and mixture of red clover (Trifolium pratense L.), perennial ryegrass (L. perenne) and timothy (Phleum pratense L.) at a ratio 60:25:15 of fresh matter, in early bloom stage of red clover (first harvest) were harvested in 2012, on 4, 7 and 14 June, respectively. The crops were cut by a mower conditioner "Kverneland Taarup 347" ("Kverneland Group", Norway) and wilted. Wilted forage was picked up and chopped using precision-chop harvester "Massey Ferguson 5130" ("Class Group", Germany) with 20 mm theoretical length of cut.

In the laboratory, the chopped alfalfa, perennial ryegrass and red clover/perennial ryegrass/timothy were ensiled in 3.0- and 0.7-L (for determination $\mathrm{pH} 3$ days after ensiling) anaerobic glass jars at a density of $0.2 \mathrm{~kg}$ $\mathrm{L}^{-1} \mathrm{DM}$. Each trial had 9 treatments (uninoculated control and 8 inoculants), with 5 micro-silos per treatment. All eight inoculants were commercial products and were applied to the forage targeting a dosage as described in Table 1.

All inoculants were diluted with distilled water prior to use to obtain a target application rate for each treatment (Table 1). The suspension obtained was applied at rate $4 \mathrm{ml} \mathrm{kg}^{-1}$ forage, when untreated control received water at rate $4 \mathrm{ml} \mathrm{kg}^{-1}$ forage. The amount of chopped forage for a given silo was weighed. Subsequently, the additives were sprayed into the fresh forage using sprayapplicators the forages thoroughly mixed by hand and then placed into the silo by hand with periodic tamping. Equipment coming into contact with the treated crop was washed and wiped with ethanol between treatments to avoid cross-contamination. Over the course of ensiling for all treatments, five samples of untreated chopped forage were taken for analysis of initial characteristics, and all inoculant solutions were analyzed for lactic acid bacteria counts on MRS (DeMan-Rogosa-Sharpe) agar (ISO 15214:1998. Microbiology of food and animal

Table 1. Inoculants used in the trials

\begin{tabular}{|c|c|c|}
\hline Product & Active ingredient & $\begin{array}{l}\text { Application } \\
{\text { cfu } \mathrm{g}^{-1} \text { forage }}\end{array}$ \\
\hline Control & Without active ingredient & - \\
\hline F10 & $\begin{array}{l}\text { Pediococcus acidilactici 33-11 NCIMB 30085, P. acidilactici 33-06 NCIMB 30086, } \\
\text { Lactobacillus plantarum LSI NCIMB 30083, L. plantarum L-256 NCIMB } 30084, \\
\text { Enterococcus faecium M74 NCIMB11181 }\end{array}$ & 100000 \\
\hline F18 & $\begin{array}{l}\text { Pediococcus acidilactici 33-11 NCIMB 30085, P. acidilactici 33-06 NCIMB 30086, } \\
\text { Lactobacillus plantarum LSI NCIMB 30083, L. plantarum L-256 NCIMB } 30084, \\
\text { Enterococcus faecium M74 NCIMB1118, xylanase }\end{array}$ & 1000000 \\
\hline F22 & $\begin{array}{l}\text { Lactococcus lactis SR } 3.54 \text { NCIMB30117, Pediococcus acidilactici 33-11 NCIMB } 30085 \text {, } \\
\text { P. acidilactici 33-06 NCIMB 30086, Enterococcus faecium M74 NCIMB1118, xylanase, } \\
\text { sodium benzoate }\end{array}$ & 200000 \\
\hline F3000 & $\begin{array}{l}\text { Lactococcus lactis SR } 3.54 \text { NCIMB30117, Pediococcus acidilactici 33-11 NCIMB 30085, } \\
\text { P. acidilactici 33-06 NCIMB 30086, Enterococcus faecium M74 NCIMB11181, } \\
\text { Lactobacillus plantarum MiLab } 393 \text { LMG } 21296\end{array}$ & 500000 \\
\hline ISA & $\begin{array}{l}\text { Enterococcus faecium, Lactobacillus plantarum, Pediococcus acidilactici, L. salivarius, } \\
\text { cellulase, hemicellulase, pentosanase, amylase }\end{array}$ & 250000 \\
\hline LD & Lactobacillus buchneri, Pediocococcus acidilactici, sodium benzoate, beta glucanase & 250000 \\
\hline $\mathrm{IBO}$ & Lactobacillus buchneri, Pediococcus pentosaceus & 100000 \\
\hline BS & Lactobacillus plantarum DSM 8862, L. plantarum DSM 8866, potassium sorbate & 300000 \\
\hline
\end{tabular}

F - Feedthech, ISA - Sil-All $4 \times 4$, LD - Lalsil Dry, IBO - Bonsilage, BS - Bio-sil Stabil; cfu - colony-forming units 
feeding stuffs - Horizontal method for the enumeration of mesophilic lactic acid bacteria - Colony count technique at $30^{\circ} \mathrm{C}$ ) (Leuschner et al., 2003). The silos were stored for 90 days at a room temperature of about $22^{\circ} \mathrm{C}$ after samples for chemical analyses had been taken.

Chemical analysis. The untreated chopped forages at ensiling and the silages at silo opening 90 days after ensiling were analyzed for the dry matter (DM) content and chemical composition, and the silages for the volatile fatty acids, lactic acid, alcohol concentration and aerobic stability as described previously by Jatkauskas et al. (2013).

Statistical analysis. Silage composition data were subjected to one-way analysis of variance for a 9 (additive) factorial arrangement of treatments within a randomized complete block design by using Proc $G L M$ of $S A S$, version 8.02 (Statistical Analysis System, 2000). Aerobic stability value for each crop type was analyzed separately by one-way analysis of variance in a randomized complete block design and where temperatures were treated as repeated measurements. Significance of the differences between the means was determined according to the least significant difference (LSD) at 0.05 probability level.

\section{Results and discussion}

Chemical composition of herbage at harvest. Differences in fermentability between grasses and legumes have been attributed to the major differences in their chemical composition, and particularly the buffering capacity (Playne, McDonald, 1996). Quantitatively, the amount of acid required to drop the original $\mathrm{pH}=6$ to a stable $\mathrm{pH}=4$ is dependent on the silages dry matter content, water soluble carbohydrates content and crude protein content. Further, forages with high dry matter content are fermented at a slower rate than forages with low dry matter because of low water activity (Rizk et al., 2005). With reference to the chemical composition, water soluble carbohydrates, crude protein content and buffering capacity values in our experiment we observed a differences between ensiled materials (Table 2). Based on the water soluble carbohydrates and crude protein content of the herbages prior to ensiling, the alfalfa forage was considered as difficult to ensile (water soluble carbohydrates concentration $15 \mathrm{~g} \mathrm{~kg}^{-1}$ fresh forage) according to the European Food Safety Authority (EFSA) opinion on silage additives guidelines (Commission Regulation (EC) No. 429/2008), while perennial ryegrass and red clover/ryegrass/timothy forages were considered to be moderately easy to ensile (water soluble carbohydrates concentration 41 and $31 \mathrm{~g} \mathrm{~kg}^{-1}$ fresh forage, respectively). The buffering capacity was considered to be typical of alfalfa, perennial ryegrass and red clover/ ryegrass/timothy herbages and ranged between 45.16 and $27.83 \mathrm{mEq} 100 \mathrm{~g}^{-1} \mathrm{DM}$. Moreover, high buffering capacity and crude protein, in combination with low water soluble carbohydrates concentration of the alfalfa meant that the ensiling properties for the alfalfa were not ideal as suggested also by Muck (2012).

Table 2. Chemical composition of herbage prior to ensiling

\begin{tabular}{lcccccc}
\hline & \multicolumn{2}{c}{ Alfalfa } & Perennial ryegrass & \multicolumn{2}{c}{ Red clover/ryegrass/timothy } \\
\cline { 2 - 7 } & mean & $\begin{array}{c}\text { standard } \\
\text { deviation }\end{array}$ & mean & $\begin{array}{c}\text { standard } \\
\text { deviation }\end{array}$ & mean & $\begin{array}{c}\text { standard } \\
\text { deviation }\end{array}$ \\
\hline Dry matter g kg-1 & 355.34 & 9.102 & 332.10 & 3.914 & 342.80 & 6.686 \\
Crude protein g kg-1 DM & 221.95 & 6.915 & 149.91 & 1.908 & 187.31 & 14.711 \\
Crude fat g kg ${ }^{-1} \mathrm{DM}$ & 31.61 & 2.819 & 26.40 & 2.875 & 19.85 & 1.580 \\
Crude fibre g kg-1 DM & 252.45 & 7.161 & 210.91 & 2.630 & 216.53 & 8.180 \\
Crude ash g kg-1 DM & 86.26 & 4.579 & 87.15 & 3.218 & 80.76 & 4.342 \\
Water soluble carbohydrates g kg ${ }^{-1} \mathrm{DM}$ & 42.20 & 3.764 & 122.98 & 3.332 & 90.87 & 2.121 \\
Acid detergent fibre g kg-1 DM & 326.42 & 18.735 & 329.02 & 11.521 & 305.22 & 5.332 \\
Neutral detergent fibre g kg $\mathrm{DM}^{-1} \mathrm{DM}$ & 376.46 & 26.808 & 435.90 & 15.561 & 413.10 & 8.769 \\
Organic matter digestibility g kg ${ }^{-1} \mathrm{DM}$ & 687.60 & 9.762 & 746.80 & 16.72 & 728.20 & 9.365 \\
pH & 6.27 & 0.078 & 5.82 & 0.066 & 5.88 & 0.040 \\
Nitrate mg kg-1 DM & 341.76 & 103.08 & 475.30 & 71.180 & 1331.97 & 101.135 \\
Buffering capacity mEq $100 \mathrm{~g} \mathrm{~g}^{-1} \mathrm{DM}$ & 45.16 & 0.608 & 27.48 & 2.091 & 27.83 & 1.875 \\
\hline
\end{tabular}

Silage characteristics; nutritional composition of silages. Some significant differences in dry matter content, crude protein, neutral detergent fiber (NDF) and water soluble carbohydrates concentrations were observed among the silages tested. The dry matter (corrected for volatiles) concentrations were higher $(P<0.05)$ for the Feedtech inoculants (F10, F3000, F18 and F22) treated alfalfa silages and for the inoculated all perennial ryegrass silages compared with the control silages. LD treated red clover/ryegrass/timothy silages had the lowest $(P<0.05)$ dry matter (corrected for volatiles) content compared to the control and other inoculated silages. Crude protein was affected by inoculation in alfalfa but not in perennial ryegrass or red clover/ryegrass/timothy silages. Only the alfalfa silage treated with inoculant products had a crude protein value statistically different from the control. Additive treated (except LD) silages had higher water soluble carbohydrates concentrations than control silages. Among the inoculated silages the water soluble carbohydrates remaining after fermentation were highest $(P<0.05)$ in the F22 treated alfalfa silage, in the F18 treated perennial ryegrass silage and in the F3000 treated red clover/ryegrass/timothy silage. The water soluble carbohydrates remaining after fermentation were lowest $(P<0.05)$ in alfalfa, perennial ryegrass and red clover/ryegrass/timothy silages treated with product LD (Table 3). 
Table 3. Nutritional composition of the silages 90 days after ensiling, $\mathrm{g} \mathrm{kg}^{-1} \mathrm{DM}$

\begin{tabular}{|c|c|c|c|c|c|c|c|c|c|c|}
\hline $\begin{array}{l}\text { Product } \\
\text { Factor No. }\end{array}$ & $\begin{array}{c}\text { Control } \\
1\end{array}$ & $\begin{array}{c}\text { F10 } \\
3\end{array}$ & $\begin{array}{c}\mathrm{F} 18 \\
5\end{array}$ & $\begin{array}{c}\mathrm{F} 22 \\
7\end{array}$ & $\begin{array}{c}\mathrm{F} 3000 \\
4\end{array}$ & $\begin{array}{l}\text { ISA } \\
2\end{array}$ & $\begin{array}{c}\text { LD } \\
6\end{array}$ & $\begin{array}{c}\mathrm{IBO} \\
8\end{array}$ & $\begin{array}{c}\mathrm{BS} \\
9\end{array}$ & Mean \\
\hline \multicolumn{11}{|c|}{ Alfalfa silages } \\
\hline $\mathrm{DM}^{*}$ & $\begin{array}{c}329.9 \\
3,4,5,7\end{array}$ & $\begin{array}{c}345.0 \\
1\end{array}$ & $\begin{array}{c}345.7 \\
1\end{array}$ & $\begin{array}{c}341.1 \\
1\end{array}$ & $\begin{array}{c}345.6 \\
1\end{array}$ & 340.3 & 335.2 & 335.8 & 338.1 & 339.6 \\
\hline Crude protein & $\begin{array}{c}188.4 \\
2,3,4,5,6,6,7,8,9\end{array}$ & $\begin{array}{c}204.2 \\
1\end{array}$ & $\begin{array}{c}219.3 \\
1,2\end{array}$ & $\underset{1}{211.8}$ & $\begin{array}{c}204.7 \\
1\end{array}$ & $\begin{array}{c}202.0 \\
1,5\end{array}$ & $\begin{array}{c}209.1 \\
1\end{array}$ & $\begin{array}{c}205.3 \\
1\end{array}$ & $\begin{array}{c}209.5 \\
1\end{array}$ & 206.0 \\
\hline $\begin{array}{l}\text { Neutral detergent } \\
\text { fibre }\end{array}$ & $\begin{array}{c}392.7 \\
5,7\end{array}$ & 382.4 & $\begin{array}{c}363.3 \\
1,4,8,9\end{array}$ & $\begin{array}{c}367.1 \\
1,4,8\end{array}$ & $\begin{array}{c}387.4 \\
5,7\end{array}$ & 379.9 & 374.4 & $\begin{array}{c}390.7 \\
5,7\end{array}$ & $\begin{array}{c}388.2 \\
5\end{array}$ & 380.7 \\
\hline $\begin{array}{l}\text { Water soluble } \\
\text { carbohydrates }\end{array}$ & $\begin{array}{l}2.66 \\
2,6,7\end{array}$ & $\begin{array}{c}3.31 \\
6,7\end{array}$ & $\begin{array}{c}4.00 \\
6,7\end{array}$ & $\begin{array}{c}6.22 \\
1,2,3,4,5,6,6,8,9\end{array}$ & $\begin{array}{c}3.36 \\
6,7\end{array}$ & $\begin{array}{l}4.23 \\
1,6,7\end{array}$ & $\begin{array}{c}1.61 \\
1,2,3,4,5,7,9\end{array}$ & $\begin{array}{c}2.79 \\
7\end{array}$ & $\begin{array}{c}3.19 \\
6,7\end{array}$ & 3.49 \\
\hline $\begin{array}{l}\text { Organic matter } \\
\text { digestibility }\end{array}$ & $\begin{array}{c}659.8 \\
5,7\end{array}$ & 666.0 & $\begin{array}{l}673.0 \\
1,2,6,8,9\end{array}$ & $\begin{array}{c}670.4 \\
1\end{array}$ & 667.2 & $664.4^{5}$ & $\begin{array}{c}663.0 \\
5\end{array}$ & $\begin{array}{c}664.8 \\
5\end{array}$ & $\begin{array}{c}663.6 \\
5\end{array}$ & 665.9 \\
\hline \multicolumn{11}{|c|}{ Perennial ryegrass silages } \\
\hline $\mathrm{DM}^{*}$ & $\begin{array}{c}316.5 \\
2,3,4,5,6,6,7,8,9\end{array}$ & $\begin{array}{c}327.6 \\
1\end{array}$ & $\begin{array}{c}327.4 \\
1\end{array}$ & $\begin{array}{c}328.8 \\
1\end{array}$ & $\begin{array}{c}326.0 \\
1\end{array}$ & $\begin{array}{c}322.3 \\
1\end{array}$ & $\begin{array}{c}324.1 \\
1\end{array}$ & $\begin{array}{c}323.0 \\
1\end{array}$ & $\begin{array}{c}325.4 \\
1\end{array}$ & 324.7 \\
\hline Crude protein & 147.1 & 147.5 & 145.7 & 146.8 & 147.2 & 147.4 & 144.7 & 143.3 & 146.0 & 146.2 \\
\hline $\begin{array}{l}\text { Neutral detergent } \\
\text { fibre }\end{array}$ & 444.8 & 441.4 & 437.9 & 433.7 & 444.4 & 438.6 & 435.1 & 444.2 & 446.7 & 440.7 \\
\hline $\begin{array}{l}\text { Water soluble } \\
\text { carbohydrates }\end{array}$ & $\begin{array}{c}26.62 \\
3,4,5\end{array}$ & $\begin{array}{l}39.19 \\
1,2,5,6,8\end{array}$ & $\begin{array}{c}53.24 \\
1,2,3,4,6,7,8,9\end{array}$ & $\begin{array}{c}33.77 \\
4,5,6,8\end{array}$ & $\begin{array}{c}45.18 \\
1,2,5,6,7,8,9\end{array}$ & $\begin{array}{c}29.11 \\
3,4,5,6\end{array}$ & $\begin{array}{c}18.39 \\
2,3,4,5,7,9\end{array}$ & $\begin{array}{c}21.64 \\
3,4,5,7\end{array}$ & $\begin{array}{c}31,39 \\
4,5,6\end{array}$ & 33.17 \\
\hline $\begin{array}{l}\text { Organic matter } \\
\text { digestibility }\end{array}$ & 722.4 & 728.0 & 733.6 & 734.8 & 729.6 & 729.4 & 726.0 & 727.8 & 727.6 & 728.0 \\
\hline \multicolumn{11}{|c|}{ Red clover/ryegrass/timothy silages } \\
\hline $\mathrm{DM}^{*}$ & $\begin{array}{c}329.2 \\
6\end{array}$ & $\begin{array}{c}335.3 \\
6\end{array}$ & $\begin{array}{c}335.0 \\
6\end{array}$ & $\begin{array}{c}327.0 \\
6\end{array}$ & $\begin{array}{c}331.0 \\
6\end{array}$ & $\begin{array}{c}333.3 \\
6\end{array}$ & $\begin{array}{c}317.5 \\
1,2,3,4,5,7,8,9\end{array}$ & $\begin{array}{c}328.4 \\
6\end{array}$ & $\begin{array}{c}331.8 \\
6\end{array}$ & 329.9 \\
\hline Crude protein & 185.4 & 186.1 & 184.4 & 190.7 & 188.2 & 190.8 & 189.1 & 182.6 & 183.5 & 186.8 \\
\hline $\begin{array}{l}\text { Neutral detergent } \\
\text { fibre }\end{array}$ & 424.7 & 421.5 & 412.1 & 411.4 & 420.5 & 414.5 & 416.9 & 420.5 & 420.2 & 418.0 \\
\hline $\begin{array}{l}\text { Water soluble } \\
\text { carbohydrates }\end{array}$ & $\begin{array}{c}9.60 \\
2,3,4,5,7,8\end{array}$ & $\begin{array}{c}19.38 \\
1,4,6,9\end{array}$ & $\begin{array}{c}16.68 \\
1,2,4,6\end{array}$ & $\begin{array}{c}18.37 \\
1,4,6\end{array}$ & $\begin{array}{c}27.50 \\
1,2,3,5,6,6,7,8,9\end{array}$ & $\begin{array}{c}22.62 \\
1,4,5,6,8,9\end{array}$ & $\begin{array}{c}8.57 \\
2,3,4,5,7,8,9\end{array}$ & $\begin{array}{c}14.69 \\
1,2,4,6\end{array}$ & $\begin{array}{c}13.39 \\
2,3,4,6\end{array}$ & 16.76 \\
\hline $\begin{array}{l}\text { Organic matter } \\
\text { digestibility }\end{array}$ & $\begin{array}{c}705.8 \\
7\end{array}$ & 717.8 & 721.6 & $\begin{array}{c}723.6 \\
1\end{array}$ & 719.2 & 716.4 & 709.2 & 715.4 & 713.6 & 715.8 \\
\hline
\end{tabular}

DM* - dry matter corrected for volatiles; F - Feedtech, ISA - Sil-All $4 \times 4$, LD - Lalsil Dry, IBO - Bonsilage, BS - Bio-sil Stabil; factor No. 1 - different $(P<0.05)$ from factor No. 2, 3, 4, 5, 6, 7, 8, 9, factor No. 2 - different $(P<0.05)$ from factor No. 1, 3, 4, $5,6,7,8,9$, etc.

Silage fermentation characteristics. The use of the inoculant additives accelerated the fermentation in alfalfa, perennial ryegrass and red clover/ryegrass/ timothy silages compared to the silages without additives. Spontaneous fermentation in the control alfalfa, perennial ryegrass and red clover/ryegrass/timothy silages produced lower concentrations of fermentation acids; however, used more sugars available in the herbages with lower $\mathrm{pH}$ drop. Higher values of $\mathrm{pH} 3$ days after ensiling in the untreated silages compared to those of the inoculanttreated silages indicate that fermentation was initiated less effectively by epiphytic microflora. The slower decline in $\mathrm{pH} 3$ days after ensiling of spontaneously fermented silage compared to inoculated silage probably reflected the low epiphytic lactic acid bacteria counts and their less efficient lactic acid production compared to a commercial strain as suggested by Davies et al. (2005). Data of the $\mathrm{pH}$ value 90 days after ensiling together with the changes in the silage water soluble carbohydrates, lactic acid, acetic acid, butyric acid and proportion of ammonia- $\mathrm{N}$ and alcohols 90 days after ensiling show that there were clear differences between the additive treatments and ensiled forages (Tables 4-6). By using inoculants, products of fermentation are shifted in alfalfa, perennial ryegrass and red clover/ryegrass/timothy silages, resulting in significantly higher lactic acid concentration and acetic acid concentration and significantly lower butyric acid, alcohols and ammoniacal nitrogen fraction. This indicates that the addition of inoculants allowed a more rapid production of lactic acid which suppresses the buffering effect of legumes and grasses as suggested by Adesogan and Salawu (2004). The largest reduction in $\mathrm{pH} 90$ days after ensiling in alfalfa, perennial ryegrass and red clover/ ryegrass/timothy silages was produced by F10, F3000, F18 and F22 products, whereas LD and IBO inoculants produced the smallest reduction in $\mathrm{pH}$ in alfalfa silages (Table 4), LD, IBO and BS - in perennial ryegrass silages (Table 5) and LD - in red clover/ryegrass/timothy silages (Table 6). 
Table 4. Fermentation characteristics of the alfalfa silages 90 days after ensiling, $\mathrm{g} \mathrm{kg}^{-1} \mathrm{DM}$

\begin{tabular}{|c|c|c|c|c|c|c|c|c|}
\hline $\begin{array}{l}\text { Treatment / } \\
\text { Factor No. }\end{array}$ & $\begin{array}{c}\mathrm{pH} \text { after } \\
3 \text { days }\end{array}$ & $\begin{array}{l}\mathrm{pH} \text { after } \\
90 \text { days }\end{array}$ & Lactate & Acetate & Butyrate & Alcohols & $\begin{array}{c}\text { Ammoniacal } \\
\text { nitrogen }\end{array}$ & DM loss* \\
\hline Control / 1 & $\begin{array}{c}6.15 \\
3,4,5,7,9\end{array}$ & $\begin{array}{c}6.02 \\
2,3,4,5,6,7,8,9\end{array}$ & $\begin{array}{c}25.63 \\
2,3,4,5,6,7,8,9\end{array}$ & $\begin{array}{c}18.40 \\
2,3,4,5,6,7,8,9\end{array}$ & $\begin{array}{c}5.30 \\
2,3,4,5,6,7,8,9\end{array}$ & $\begin{array}{c}7.79 \\
2,3,4,5,6,7,8,9\end{array}$ & $\begin{array}{c}96.07 \\
2,3,4,5,6,7,8,9\end{array}$ & $\begin{array}{c}84.48 \\
2,3,4,5,6,6,7,8,9\end{array}$ \\
\hline $\mathrm{F} 10 / 3$ & $\begin{array}{c}5.29 \\
1,2,4,6,8,9\end{array}$ & $\begin{array}{l}4.94 \\
1,4,6,7\end{array}$ & $\begin{array}{c}55.89 \\
1\end{array}$ & $\begin{array}{c}23.80 \\
1,6,9\end{array}$ & $\begin{array}{c}1.00 \\
1,6\end{array}$ & $\begin{array}{c}2.71 \\
1,2\end{array}$ & $\begin{array}{c}56.16 \\
1\end{array}$ & $\begin{array}{c}41.15 \\
1\end{array}$ \\
\hline F18 / 5 & $\begin{array}{c}5.43 \\
1,2,6,8,9\end{array}$ & $\begin{array}{l}4.88 \\
1,6,8,9\end{array}$ & $\begin{array}{c}59.38 \\
1\end{array}$ & $\begin{array}{c}23.80 \\
1,6,9\end{array}$ & $\begin{array}{c}0.66 \\
1,6\end{array}$ & $\begin{array}{c}3.24 \\
1,2\end{array}$ & $\begin{array}{c}52.46 \\
1\end{array}$ & $\begin{array}{c}39.04 \\
1,6\end{array}$ \\
\hline F22 / 7 & $\begin{array}{c}5.36 \\
1,2,4,6,8,9\end{array}$ & $\begin{array}{c}4.85 \\
1,3,6,8,9\end{array}$ & $\begin{array}{c}56.23 \\
1\end{array}$ & $\begin{array}{c}24.31 \\
1,6,8,9\end{array}$ & $\begin{array}{c}0.96 \\
1,6\end{array}$ & $\begin{array}{c}2.78 \\
1,2\end{array}$ & $\begin{array}{c}60.57 \\
1\end{array}$ & $\begin{array}{c}48.70 \\
1\end{array}$ \\
\hline F3000 / 4 & $\begin{array}{c}5.56 \\
1,2,3,6,7,8,9\end{array}$ & $\begin{array}{c}4.86 \\
1,2,3,6,8,9\end{array}$ & $\begin{array}{c}54.46 \\
1\end{array}$ & $\begin{array}{c}24.61 \\
1,6,8,9\end{array}$ & $\begin{array}{c}1.04 \\
1,6\end{array}$ & $\begin{array}{c}3.31 \\
1,2\end{array}$ & $\begin{array}{c}57.89 \\
1\end{array}$ & $\begin{array}{c}40.74 \\
1\end{array}$ \\
\hline ISA / 2 & $\begin{array}{l}6.02 \\
3,4,5,7\end{array}$ & $\begin{array}{l}4.93 \\
1,4,6,8\end{array}$ & $\begin{array}{c}49.97 \\
1\end{array}$ & $\begin{array}{c}24.00 \\
1,6,8,9\end{array}$ & $\underset{1}{2.11}$ & $\begin{array}{c}5.59 \\
1,3,4,5,7,8\end{array}$ & $\begin{array}{c}59.91 \\
1\end{array}$ & $\begin{array}{c}48.93 \\
1\end{array}$ \\
\hline $\mathrm{LD} / 6$ & $\begin{array}{l}6.07 \\
3,4,5,7\end{array}$ & $\begin{array}{c}5.16 \\
1,2,3,4,5,7,8,9\end{array}$ & $\begin{array}{c}48.47 \\
1\end{array}$ & $\begin{array}{c}31.39 \\
1,2,3,4,5,7\end{array}$ & $\begin{array}{c}3.48 \\
1,3,4,5,7\end{array}$ & $\begin{array}{c}4.19 \\
1\end{array}$ & $\begin{array}{c}57.14 \\
1\end{array}$ & $\begin{array}{c}63.96 \\
1\end{array}$ \\
\hline $\mathrm{IBO} / 8$ & $\begin{array}{l}5.98 \\
3,4,5,7\end{array}$ & $\begin{array}{c}5.01 \\
1,3,4,5,6,7\end{array}$ & $\begin{array}{c}47.78 \\
1\end{array}$ & $\begin{array}{c}29.66 \\
1,2,4,7\end{array}$ & $\begin{array}{c}1.75 \\
1\end{array}$ & $\begin{array}{c}3.25 \\
1,2\end{array}$ & $\begin{array}{c}58.44 \\
1\end{array}$ & $\begin{array}{c}61.69 \\
1\end{array}$ \\
\hline $\mathrm{BS} / 9$ & $\begin{array}{c}5.90 \\
1,3,4,5,7\end{array}$ & $\begin{array}{c}4.98 \\
1,4,5,6,7\end{array}$ & $\begin{array}{c}54.53 \\
1\end{array}$ & $\begin{array}{c}31.54 \\
1,2,3,4,5,7\end{array}$ & $\begin{array}{c}2.18 \\
1\end{array}$ & $\begin{array}{c}3.73 \\
1,2\end{array}$ & $\begin{array}{c}60.08 \\
1\end{array}$ & $\begin{array}{c}50.71 \\
1\end{array}$ \\
\hline Mean & 5.75 & 5.07 & 50.3 & 25.72 & 2.05 & 4.07 & 62.08 & 53.27 \\
\hline
\end{tabular}

Note. Explanations under Table 3; DM loss* - dry matter loss corrected for volatiles.

In alfalfa, perennial ryegrass and red clover/ ryegrass/timothy silages the inoculated treatments produced larger $(P<0.05)$ lactate concentrations than the uninoculated treatment. Perennial ryegrass inoculated with
F18 and F22 had greater lactate concentrations than other inoculated silages (Table 5). Perennial ryegrass silages inoculated with LD, F22 and IBO had higher $(P<0.05)$ acetate concentrations than the control silages.

Table 5. Fermentation characteristics of the perennial ryegrass silages 90 days after ensiling, $\mathrm{g} \mathrm{kg}^{-1} \mathrm{DM}$

\begin{tabular}{|c|c|c|c|c|c|c|c|c|}
\hline $\begin{array}{l}\text { Treatment / } \\
\text { Factor No. }\end{array}$ & $\begin{array}{c}\mathrm{pH} \text { after } \\
3 \text { days }\end{array}$ & $\begin{array}{l}\mathrm{pH} \text { after } \\
90 \text { days }\end{array}$ & Lactate & Acetate & Butyrate & Alcohols & $\begin{array}{c}\text { Ammoniacal } \\
\text { nitrogen }\end{array}$ & $\begin{array}{c}\text { DM } \\
\text { loss* }\end{array}$ \\
\hline \multirow[t]{2}{*}{ Control / 1} & 4.71 & 4.46 & 38.68 & 25.03 & 2.58 & 6.10 & 32.85 & 56.18 \\
\hline & $2,3,4,5,6,7,8,9$ & $2,3,4,5,6,7,8,9$ & $2,3,4,5,6,7,8,9$ & $6,7,8$ & $2,3,4,5,7$ & $2,3,4,5,7$ & $2,3,4,5,7,9$ & $2,3,4,5,6,7,8,9$ \\
\hline \multirow[t]{2}{*}{$\mathrm{F} 10 / 3$} & 4.31 & 4.04 & 64.95 & 29.10 & 0.54 & 3.29 & 18.62 & 29.44 \\
\hline & $1,6,7$ & $1,6,8,9$ & $1,5,7$ & & 1 & $1,6,8,9$ & $1,6,8$ & 1 \\
\hline \multirow[t]{2}{*}{ F18 / 5} & 3.76 & 4.01 & 87.63 & 32.87 & 0.28 & 3.76 & 20.72 & 24.79 \\
\hline & $1,6,8,9$ & $1,6,8,9$ & $1,2,3,4,6,8$ & & 1 & $1,6,8,9$ & $1,6,8$ & 1,6 \\
\hline \multirow[t]{2}{*}{ F22 / 7} & 3.87 & 4.00 & 97.84 & 33.84 & 0.85 & 3.87 & 18.27 & 22.67 \\
\hline & $1,6,8,9$ & $1,6,8,9$ & $1,2,3,4,6,8,9$ & 1 & 1 & $1,6,8,9$ & $1,6,8$ & 1,6 \\
\hline \multirow{2}{*}{ F3000 / 4} & 4.25 & 4.04 & 72.73 & 27.69 & 0.70 & 3.25 & 22.48 & 32.51 \\
\hline & $1,2,6,8,9$ & $1,6,8,9$ & $1,5,7$ & 6 & 1 & $1,6,8,9$ & 1,6 & 1 \\
\hline \multirow[t]{2}{*}{ ISA / 2} & 4.40 & 4.05 & 64.04 & 30.11 & 0.09 & 3.02 & 19.45 & 38.87 \\
\hline & $1,4,5,7$ & $1,6,8,9$ & $1,5,7$ & & & $1,6,8,9$ & $1,6,8$ & 1 \\
\hline \multirow[t]{2}{*}{$\mathrm{LD} / 6$} & 6.35 & 4.26 & 64.30 & 36.72 & 1.22 & 6.35 & 30.56 & 44.07 \\
\hline & $2,3,4,5,7$ & $1,2,3,4,5,7,8,9$ & $1,5,7$ & 1,4 & & $2,3,4,5,7$ & $12,3,4,5,7,9$ & $1,5,7$ \\
\hline \multirow[t]{2}{*}{ IBO / 8} & 5.50 & 4.20 & 64.67 & 33.45 & 1.67 & 5.50 & 27.79 & 38.16 \\
\hline & $2,3,4,5,7$ & $1,2,3,4,5,6,7$ & $1,5,7$ & 1 & & $2,3,4,5,7$ & $2,3,5,7$ & 1 \\
\hline \multirow[t]{2}{*}{$\mathrm{BS} / 9$} & 5.42 & 4.16 & 77.36 & 31.04 & 1.41 & 5.42 & 23.15 & 32.96 \\
\hline & $2,3,4,5,7$ & $1,2,3,4,5,6,7$ & 1,7 & & & $2,3,4,5,7$ & 1,6 & 1 \\
\hline Mean & 4.51 & 4.13 & 70.24 & 31.09 & 1.10 & 4.51 & 23.76 & 35.53 \\
\hline
\end{tabular}

Note. Explanations under Table 3; DM loss* - dry matter loss corrected for volatiles.

Red clover/ryegrass/timothy silages inoculated with LD had lower lactate concentrations $(P<0.05)$ compared with silages inoculated with products F10, F3000, F18 and F22 treated silages, but produced more $(P<0.05)$ acetate than control silage and other inoculated silages (Table 6). Red clover/ryegrass/timothy silages inoculated with LD produced more $(P<0.05)$ acetate than control silage and other inoculated silages. Lower $\mathrm{pH}$ and higher lactate content in silage fermentation with homofermentative lactic acid bacteria (F10, F3000, F18 and F22) are consistent with summaries of studies by Kung et al. (2003) when such inoculants were successful. Heterofermentative lactic acid bacteria are less efficient in producing lactic acid than homofermentative lactic acid bacteria usually resulting in more acetic acid, higher $\mathrm{pH}$, higher ethanol and higher dry matter losses (Filya et al., 2007).

Red clover/ryegrass/timothy silages inoculated with LD produced more $(P<0.05)$ acetate than control silage and other inoculated silages. The high ethanol concentration of the control silage probably also resulted from a clostridial fermentation, as suggested by Muck (2012). In alfalfa silage, ammonia-N fraction was lower for inoculant treated silages compared to the control silage. LD and IBO silages had similar ammoniacial nitrogen fraction concentration compared to the control silage, 
Table 6. Fermentation characteristics of the red clover/ryegrass/timothy silages 90 days after ensiling, $\mathrm{g} \mathrm{kg}^{-1} \mathrm{DM}$

\begin{tabular}{|c|c|c|c|c|c|c|c|c|}
\hline $\begin{array}{l}\text { Treatment / } \\
\text { Factor No. }\end{array}$ & $\begin{array}{l}\mathrm{pH} \text { after } \\
3 \text { days }\end{array}$ & $\begin{array}{l}\mathrm{pH} \text { after } \\
90 \text { days }\end{array}$ & Lactate & Acetate & Butyrate & Alcohols & $\begin{array}{c}\text { Ammoniacal } \\
\text { nitrogen }\end{array}$ & DM loss* \\
\hline Control / 1 & $\begin{array}{c}4.37 \\
2,3,4,5,6,6,7,8,9\end{array}$ & $\begin{array}{c}4.37 \\
2,3,4,5,6,6,7,8,9\end{array}$ & $\begin{array}{c}37.10 \\
2,3,4,5,6,6,9,9\end{array}$ & $\begin{array}{c}24.25 \\
2,3,4,5,6,7,8,9\end{array}$ & $\begin{array}{c}2.56 \\
7\end{array}$ & $\begin{array}{c}6.32 \\
2,3,4,5,6,7,8,9\end{array}$ & $\begin{array}{l}32.49 \\
2,3,4,5,7,9\end{array}$ & $\begin{array}{c}58.77 \\
2,3,4,5,6,9\end{array}$ \\
\hline $\mathrm{F} 10 / 3$ & $\begin{array}{c}4.26 \\
1,2,6,7,8,9\end{array}$ & $\begin{array}{c}4.06 \\
1,2,5,6,7,8,9\end{array}$ & $\begin{array}{c}57.94 \\
1,6,8\end{array}$ & $\begin{array}{l}16.58 \\
1,5,6,8,9\end{array}$ & 0.41 & $\begin{array}{l}3.57 \\
1,2,6,8,9\end{array}$ & $\begin{array}{c}16.26 \\
1,4,5,6,7,8,8,9\end{array}$ & $\begin{array}{c}32.30 \\
1,6,7\end{array}$ \\
\hline $\mathrm{F} 18 / 5$ & $\begin{array}{l}4.31 \\
1,2,6,7,9\end{array}$ & $\begin{array}{c}4,13 \\
1,2,3,4,6,7\end{array}$ & $\begin{array}{l}51.56 \\
1,6,7,8\end{array}$ & $\begin{array}{c}13.12 \\
1,2,3,4,6,7,8,9\end{array}$ & 0.61 & $\begin{array}{c}4.13 \\
1,6,9\end{array}$ & $\begin{array}{c}25.08 \\
1,2,3,6\end{array}$ & $\begin{array}{c}29.69 \\
1,6,7\end{array}$ \\
\hline $\mathrm{F} 22 / 7$ & $\begin{array}{c}4.17 \\
1,2,3,5,6,6,9\end{array}$ & $\begin{array}{c}4.00 \\
1,2,3,5,6,8,9\end{array}$ & $\begin{array}{l}60.44 \\
1,5,6,8,9\end{array}$ & $\begin{array}{l}15.89 \\
1,5,6,8,9\end{array}$ & $\begin{array}{c}0.18 \\
1\end{array}$ & $\begin{array}{r}4.09 \\
1,6,9\end{array}$ & $\begin{array}{c}25.85 \\
1,2,3,6\end{array}$ & $\begin{array}{c}51.51 \\
2,3,5,6\end{array}$ \\
\hline F3000 / 4 & $\begin{array}{l}4.30 \\
1,2,6,7,9\end{array}$ & $\begin{array}{c}4.01 \\
1,2,5,5,6,8,9\end{array}$ & $\begin{array}{c}58.77 \\
1,6,8\end{array}$ & $\begin{array}{l}16.13 \\
1,5,6,8,9\end{array}$ & 0.89 & $\begin{array}{c}3.97 \\
1,6,9\end{array}$ & $\begin{array}{c}22.84 \\
1,3,6\end{array}$ & $\begin{array}{c}39.99 \\
1,6\end{array}$ \\
\hline ISA / 2 & $\begin{array}{l}4.41 \\
1,3,4,5,7\end{array}$ & $\begin{array}{c}4.19 \\
1,3,4,5,6,6,7,8\end{array}$ & $\begin{array}{c}54.50 \\
1,6,8\end{array}$ & $\begin{array}{c}17.51 \\
1,5,6\end{array}$ & 0.81 & $\begin{array}{l}4.53 \\
1,3,6,9\end{array}$ & $\begin{array}{c}18.33 \\
1,5,6,7,8,9\end{array}$ & $\begin{array}{c}33.55 \\
1,6,7\end{array}$ \\
\hline $\mathrm{LD} / 6$ & $\begin{array}{l}4.41 \\
1,3,4,5,5\end{array}$ & $\begin{array}{c}4.29 \\
1,2,3,4,5,7,8,9\end{array}$ & $\begin{array}{c}26.30 \\
1,2,3,4,5,7,8,9\end{array}$ & $\begin{array}{c}32.06 \\
1,2,3,4,5,7,8,9\end{array}$ & 2.17 & $\begin{array}{c}7.16 \\
1,2,3,4,5,7,8,9\end{array}$ & $\begin{array}{c}33.15 \\
2,3,4,5,7,9\end{array}$ & $\begin{array}{c}81.98 \\
1,2,3,4,5,7,8,9\end{array}$ \\
\hline $\mathrm{IBO} / 8$ & $\begin{array}{l}4.36 \\
1,3,7,9\end{array}$ & $\begin{array}{c}4.13 \\
1,2,3,4,6,7\end{array}$ & $\begin{array}{c}42.29 \\
2,3,4,5,6,6,7,9\end{array}$ & $\begin{array}{l}18.75 \\
1,3,4,5,6,7\end{array}$ & 1.00 & $\begin{array}{l}4.41 \\
1,3,6,9\end{array}$ & $\begin{array}{c}29.35 \\
2,3\end{array}$ & $\begin{array}{c}46.32 \\
6\end{array}$ \\
\hline $\mathrm{BS} / 9$ & $\begin{array}{c}4,45 \\
1,3,4,5,7,8\end{array}$ & $\begin{array}{l}4.15 \\
1,3,4,6,7\end{array}$ & $\begin{array}{l}50.32 \\
1,6,7,8\end{array}$ & $\begin{array}{c}18.97 \\
1,3,4,5,6,7\end{array}$ & 0.46 & $\begin{array}{c}5.60 \\
1,2,3,4,5,6,7,8\end{array}$ & $\begin{array}{r}25.17 \\
1,2,3,6\end{array}$ & $\underset{1,6}{41.34}$ \\
\hline Mean & 4.38 & 4.15 & 48.80 & 19.25 & 1.01 & 4.86 & 25.39 & 46.16 \\
\hline
\end{tabular}

Note. Explanations under Table 3; DM loss* - dry matter loss corrected for volatiles.

when inoculants F10, F3000, F18, F22 and ISA silages had lower $(P<0.05)$ ammoniacial nitrogen fraction than the control silage. Red clover/ryegrass/timothy silages inoculated with LD had similar ammonia-N fraction to the control silage, while inoculants F10, F3000, F18, F22 and ISA silages had lower $(P<0.05)$ ammonia-N fraction than the control silage. In alfalfa silages dry matter loss values were lower $(P<0.05)$ for all inoculant treatments and no significant differences were between the inoculant treatments. In perennial ryegrass and red clover/ryegrass/ timothy the lowest dry matter loss was achieved in silages treated with F10, F18 and F22 and the highest dry matter loss were obtained in silages treated with LD L. buchnery is a slow-growing bacterial species; the $\mathrm{pH}$ might drop slowly, allowing enterobacteria more time for protein breakdown, resulting in higher ammonia-N levels and dry matter losses (Schmidt et al., 2009).

Aerobic stabilityof silages. The data presented in this paper show that all eight products used for inoculation increased the aerobic stability of alfalfa, perennial ryegrass and red clover/ryegrass/timothy silages compared with the control silage (Fig.). Every inoculant improved aerobic stability significantly $(P<0.05)$ when compared individually to the uninoculated control. The aerobic stability of inoculated alfalfa silages was improved: by $60 \mathrm{~h}$ for ISA, by $84 \mathrm{~h}$ for F10, by $114 \mathrm{~h}$ for F3000 and F18, by $150 \mathrm{~h}$ for LD, by $174 \mathrm{~h}$, for IBO and by $132 \mathrm{~h}$ for BS compared to the control silage. Aerobic stability of alfalfa silage treated with product F22 was improved dramatically. The aerobic stability of inoculated perennial ryegrass silages was improved: by $18 \mathrm{~h}$ for ISA, by $66 \mathrm{~h}$ for F10 and F3000, by $48 \mathrm{~h}$ for F18, by $162 \mathrm{~h}$ for F22, by $96 \mathrm{~h}$ for IBO and by $126 \mathrm{~h}$ for BS compared to control silage. Aerobic stability of the perennial ryegrass silage treated with product LD was improved dramatically. The aerobic stability of inoculated red clover/ryegrass/ timothy silages was improved: by $24 \mathrm{~h}$ for ISA, by $72 \mathrm{~h}$ for F10, by $60 \mathrm{~h}$ for F3000, by $90 \mathrm{~h}$ for F18, by $138 \mathrm{~h}$ for
LD, by $132 \mathrm{~h}$ for F22, by $84 \mathrm{~h}$ for IBO and $102 \mathrm{~h}$ for BS compared to the control silage.

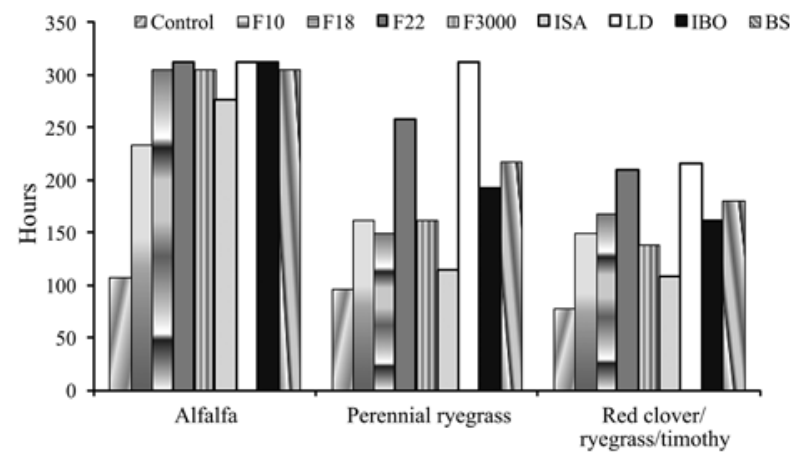

Note. Aerobic stability was considered compromised with silage temperature increased $3^{\circ} \mathrm{C}$ above ambient temperature; F - Feedtech, ISA - Sil-All $4 \times 4$, LD - Lalsil Dry, IBO Bonsilage, BS - Bio-sil Stabil.

Figure. Hours after oxygen exposure when aerobic stability was compromised

Ratanapibulsawat et al. (2005) and Broberg et al. (2007) have isolated lactic acid bacteria strains from silages that produce inhibitory activity against a variety of undesirable bacterial species and improve aerobic stability of the silages. Prema et al. (2010) indicated that L. plantarum strain can inhibit a wide range of mould species common to silage. Reis et al. (2005) provide more definitive evidence for the existence of certain lactic acid bacteria strains with the power to inhibit yeast and moulds growth, to control temperature during aerobic exposure and to improve aerobic stability. The improvement of aerobic stability was observed in a timothy/meadow fescue silage by using L. buchneri or sodium benzoate in combination with L. plantarum (Jaakkola et al., 2010). Auerbach and Weiss (2012) concluded that inoculants containing L. plantarum, L. buchnery, sodium benzoate or potassium sorbate affected dry matter losses, $\mathrm{pH}$ and 
fermentation pattern, but no effect was found on aerobic stability.

\section{Conclusions}

1. Homo and hetero lactic acid bacteria based inoculants or blends of lactic acid bacteria and other components changed fermentation profile of alfalfa, perennial ryegrass and red clover/ryegrass/timothy silages, ensiled in mini silos, by decreased $\mathrm{pH}$ value, shifted lacate:acetate ratio, reduced ammonia-N and butyrate formation.

2. Inoculation significantly improved the fermentation quality of the alfalfa, perennial ryegrass and red clover/ryegrass/timothy silage, resulting in lower dry matter losses and better aerobic stability.

3. In particular, Feedtech (F) and Sil-All $4 \times 4$ (ISA) inoculants consistently improved silage quality and were effective in reducing dry matter losses and in protecting the silages against proteolysis compared with spontaneously fermented silage and other inoculant treatments.

Received 18062014 Accepted 28112014

\section{References}

Adesogan A. T., Salawu M. B. 2004. Effect of applying formic acid, heterolactic bacteria or homolactic and heterolactic bacteria on the fermentation of bi-crops of peas and wheat. Journal of the Science of Food and Agriculture, 84: 983992

http://dx.doi.org/10.1002/jsfa.1745

Auerbach H., Weiss K. 2012. The effect of different types of silage additives on dry matter losses, fermentation pattern, volatile organic compounds and aerobic stability of sorghum silage. $16^{\text {th }}$ International Silage Conference. Hameenlinna, Finland, p. 418-419

Broberg A., Jacobsson K., Strom K., Schnurer J. 2007. Metabolite profiles of lactic acid bacteria in grass silage. Applied and Environmental Microbiology, 73: 5547-5552 http://dx.doi.org/10.1128/AEM.02939-06

Commission Regulation EC No. 429/2008. European Food Safety Authority. 2008. http://ec.europa.eu/food/food/ animalnutrition/feedadditives/guidelines_en.htm

Contreras-Govea F. E., Muck R. E., Broderick G. A., Weimer P. J. 2009. Lactobacillus plantarum MTD/1, its impact on silage and in vitro rumen fermentation. $15^{\text {th }}$ International Silage Conference. Madison, USA, p. 57-58

Davies D. R., Theodorou M. K., Kingston-Smith A. H., Merry R. J. 2005. Advances in silage quality in the $21^{\text {st }}$ century. $14^{\text {th }}$ International Silage Conference. Belfast, Northern Ireland, p. 121-133

Filya I., Muck R. E., Contreras-Govea F. E. 2007. Inoculant effects on alfalfa silage: fermentation products and nutritive value. Journal of Dairy Science, 90: 5108-5114 http://dx.doi.org/10.3168/jds.2006-877

Jaakkola S., Saarisalo E., Heikkila T. 2010. Aerobic stability and fermentation quality of round bale silage treated with inoculants or propionic acid. $23^{\text {nd }}$ general meeting of the European Grassland Federation. Kiel, Germany, p. 503-505
Jatkauskas J., Vrotniakiene V., Ohlsson C., Lund B. 2013. The effects of three silage inoculants on aerobic stability in grass, clover grass, lucerne and maize silages. Agricultural and Food Science, 22: 137-144

Kleinschmit D. H., Schmidt R. J., Kung Jr. L. 2005. The effects of various antifungal additives on the fermentation and aerobic stability of corn silage. Journal of Dairy Science, 88: 2130-2139 http://dx.doi.org/10.3168/jds.S0022-0302(05)72889-7

Kung L., Stokes M., Lin C. 2003. Silage additives. Buxton D. R. et al. (eds.). Silage science and technology, p. 305-360

Leuschner R. G. K., Jan B., Paul S. J., Ross P. R., Stanton C. 2003. Enumeration of probiotic pediococci in animal feed: interlaboratory study. Journal of AOAC International, 86 (4): 791-801

Muck R. 2012. Microbiology of ensiling. $16^{\text {th }}$ International Silage Conference. Hameenlinna, Finland, p. 75-86

Playne M. K., McDonald P. 1996. The buffering constituents of herbage and of silage. Journal of the Science of Food and Agriculture, 17: 264-268

http://dx.doi.org/10.1002/jsfa.2740170609

Prema P., Smila D., Palavesam A., Immanuel G. 2010. Production and characterization of an antifungal compound (3-phenyllactic acid) produced by Lactobacillus plantarum strain. Food and Bioprocess Technology, 3: 379-386 http://dx.doi.org/10.1007/s11947-008-0127-1

Ratanapibulsawat C., Kroujkaew P., Sadahiro O., Nitisinprasert S., Chatinan R., Pumrussiri K., Sunee N. 2005. Screening and characterization of lactic acid bacteria producing antimicrobial substance against Staphylococcus aureus. Kasetsart Journal (Natural Sciences), 39: 284-293

Reis R., Almeida E., Siqueira G., Bernardes E. R., Janusckiewicz E. 2005. Microbial changes and aerobic stability in high moisture maize silages inoculated with Lactobacillus buchnery. $14^{\text {th }}$ International Silage Conference. Belfast, Northen Ireland, p. 223

Rizk C., Mustafa A. F., Phillip L. E. 2005. Effects of inoculation of high dry matter alfalfa silage on ensiling characteristics, ruminal nutrient degradability and dairy cow performance. Journal of the Science of Food and Agriculture, 85: 743750

http://dx.doi.org/10.1002/jsfa.2034

Schmidt R., Hu W., Mills J., Kung L. 2009. The development of lactic acid bacteria and Lactobacillus buchnery and their effects on the fermentation of alfalfa silage. Journal of Dairy Science, 92: 5005-5010 http://dx.doi.org/10.3168/jds.2008-1701

Sucu E., Filya I. 2006. Effects of homofermentative lactic acid bacterial inoculants on the fermentation and aerobic stability characteristics of low dry matter corn silages. Turkish Journal of Veterinary and Animal Sciences, 30 (1): 83-88

Statistical Analysis System. 2000. User's guide: Statistics, version 8.02. SAS Institute Inc. Cary, USA

Weib K., Gerlach K., Sudekum K.-H. 2011. Fluchtige Substanzen in Maissilagen in Abhangigkeit von Silierbedingungen und aerober Laerungsdauer. Speyer, Germany, p. 534-540 (in German) 
ISSN 1392-3196 / e-ISSN 2335-8947

Zemdirbyste-Agriculture, vol. 102, No. 1 (2015), p. 95-102

DOI $10.13080 / \mathrm{z}-\mathrm{a} .2015 .102 .012$

\title{
İvairių inokuliantų ịtaka liucernụ, svidrių ir raudonųjų dobilų, svidrių bei motiejukų mišinio siloso savybėms
}

\author{
J. Jatkauskas ${ }^{1}$, V. Vrotniakiené ${ }^{1}$, A. Lanckriet ${ }^{2}$ \\ ${ }^{1}$ Lietuvos sveikatos mokslų universiteto Gyvulininkystès institutas \\ ${ }^{2}$ DeLaval NV, Belgija
}

\section{Santrauka}

Siekiant nustatyti ịvairių inokuliantų ịtaką siloso mitybinei sudėčiai, pH rodikliui, lakiuju riebalu rūgščiu kiekiui, sausųjų medžiagų nuostoliams ir aerobiniam stabilumui, 2012-2013 m. buvo atlikti laboratoriniai in vitro eksperimentai, naudojant 3 L laboratorines siloso talpas. Tirtas trijų rūšių silosas: liucernų, daugiamečių svidrių ir raudonųjų dobilų, svidrių bei motiejukų mišinio. Kiekvienos žolès silosas buvo pagamintas be jokių priedų (kontrolinis variantas), su keturių Feedtech (F) produktų (F10, F18, F22, F3000) ir produktų Sil-All $4 \times 4$ (ISA), Lalsil Dry (LD), Bonsilage (IBO) bei Bio-sil Stabil (BS) priedais. Žaliava siloso talpose buvo suslègta tiek, kad 1 litre būtų $0,2 \mathrm{~kg}$ sausujjų medžiagų. Po 90 dienų laikymo $20{ }^{\circ} \mathrm{C}$ temperatūroje siloso talpos buvo atidarytos ir paimti mėginiai sausųjų medžiagų bei jų nuostolių, pH rodikliui, lakiụjų riebalų rūgščių, amoniakinio azoto, alkoholių kiekiui, cheminei suděčiai ir aerobiniam stabilumui nustatyti. Visi aštuoni mikrobiniai inokuliantai turejjo teigiamos įtakos visų rūšių siloso fermentacijos rodikliams ir galutinei siloso kokybei, nes esmingai sumažino pH rodiklį, amoniakinio azoto kiekị bei sausujų medžiagų nuostolius ir esmingai padidino pieno rūgšties kiekị, lyginant su kontrolinio siloso rodikliais. Liucernos ir daugiametės svidrès siloso, pagaminto su inokuliantais F18, F22, F3000 ir ISA, pH rodiklis buvo esmingai mažesnis nei siloso, pagaminto su inokuliantais F10, LD, IBO ir BS. Raudonųjų dobilų, svidrių bei motiejukų mišinio siloso, pagaminto su inokuliantais F10, F22, ir F3000, pH rodiklis buvo esmingai mažesnis nei siloso, pagaminto su inokuliantais F18, ISA, LD, IBO ir BS. Daugiametès svidrès su inokuliantais F18 ir F22 silose pieno rūgšties buvo esmingai daugiau nei silose su inokuliantais F10, F3000, ISA ir LD, o raudonujų dobilų, svidrių bei motiejukų mišinio silose su inokuliantais F10, F22, F3000 ir ISA - esmingai daugiau nei silose su inokuliantais F18, LD, IBO ir BS. Sausujų medžiagų nuostoliai buvo esmingai mažesni daugiametės svidrès silose su F10, F18, F22 ir F3000 nei silose su ISA, LD ir IBO, o raudonujų dobilų, svidrių bei motiejukų mišinio silose su inokuliantais F10, F18, F3000 ir ISA - esmingai mažesni nei silose su F22, LD, IBO ir BS. Inokuliantų priedas liucernos siloso aerobinị stabilumą pagerino 126-204 valandomis, daugiametės svidrès siloso - 18-216 valandomis, raudonujų dobilų, svidrių bei motiejukų mišinio siloso - 30-138 valandomis. Tyrimai parodè, kad pieno rūgšties bakteriju inokuliantai esmingai gerina vidutiniškai ir sunkiai silosuojamu augalu siloso kokybę. Iš visų tirtų buvo efektyviausi ir siloso kokybę nuosekliai gerino inokuliantai Feedtech (F) ir Sil-All $4 \times$ 4 (ISA).

Reikšminiai žodžiai: aerobinis stabilumas, fermentacija, pieno rūgšties bakterijos. 